\title{
Mood Disorders and How They are Related to Death Anxiety for Some Infirmaries in Baghdad
}

\author{
Aya Muayad Sadoon', Hawraa Mohammed Ali Hassan² \\ ${ }^{1}$ The General Directorate of DhiQar Education/Sayed Dekhel, \\ ${ }^{2}$ The General Directorate of DhiQar Education/Al-Islah Department
}

\begin{abstract}
The subject of mood disorders and death anxiety in the elderly is considered one of the topics that have been addressed only slightly and in limited ways in relation to general trends and psychological research in other subjects in terms of quality and quantity. As well as if there is a statistically significant effect of sex variables, the sample of the study consisted of all the elderly who were able to respond to the researcher, which amounted to (110) elderly people, including (65) males and (45) females. We collected demographic information about the elderly, as well as translating the mood disorders scale, as well as the arithmetic averages, percentages, deviations and the Pearson correlation coefficient were used. To reach the results of the study, a number of results were reached. The suffering of elderly people living in nursing homes in the capital, Baghdad, from a high level of mood disorders. The suffering of the elderly residing in the nursing homes of the capital, Baghdad, from a moderate feeling in the level of death anxiety. There are statistically significant differences at the level of (0.05) between the elderly on the measures of mood disorders and death anxiety that are related to the gender variable and return to the benefit of females. There is no statistically significant relationship between mood disorders and death anxiety among the study sample.
\end{abstract}

Keywords: Death anxiety, Mood disorders, infirmaries, Baghdad.

\section{Introduction}

The subject of mood disorders and death anxiety in the elderly is considered one of the topics that have been addressed only sparingly - to the best of the researcher's knowledge - and in limited ways, and it can be said since the late last century that the phenomenon of aging has become one of the essential axes within the field of modern psychological research and its topic has attracted greater attention among Researchers since ever since this stage carries the characteristics and components of the life stages that preceded it ${ }^{1}$. It is not a coincidence that the issue of the elderly takes center stage in the interests of scholars, because each of us humans is getting old, and with age, changes in his daily life undergo changes, some of which are biological, some are social, and some are psychological, so the individual turns into a battlefield In it the different forces and he has to reconcile between them and the more he succeeds in that, his psychological life proceeds together and balanced, and the more the opposite is realized by despair and despair, and he develops a sense of dispersed self that prepares him for a life full of turmoil and the result of that is either mental or psychological disorder ${ }^{3}$. Among the stable facts is that mood disorders and the opposing symptoms that accompany them, such as depression or mania, or both, are considered among the common disorders of the age and perhaps more prevalent for the elderly, as the individual among them feels that he lives in an age that he does not understand and differs from the era in which he lived and that this feeling leads By his lack of adaptation to the new era, which may affect his outlook on life by changing his mood ${ }^{3}$. Mood disorders are disorders that result from failed attempts to resolve unconscious emotional crises, and that this failure to adapt leads to the accumulation of feelings of tension and anxiety in the individual, and we may find that these disorders are associated with anxiety disorder, which is a common symptom ${ }^{4}$ of mental disorders and takes several types, including normal or objective anxiety, and anxiety Chronic acute and condition anxiety and trait anxiety etc.

Among the types of anxiety that the elderly are 
subjected to in their lives is the so-called death anxiety. This type of anxiety is a state of intellectual disturbance - mood in front of the imminent danger to the body and raises more questions and contradictory problems due to the privacy imposed on the human being required by the laws of nature, and the elderly by their nature live An unconscious state of dying phobia, which causes them to have tremendous anxiety and causes a paradoxical disturbance in their behavior. Despite the importance of this approach in diagnosis and treatment, it receives sufficient attention from Arab and Muslim researchers.

For this reason, many researchers have unanimously agreed that anxiety about death is the basis of psychological disorder, which is the origin of mental illness. Rather, some of them have argued that the situation differs in different moods, for example that the person with a bloody mood is longer than the bile and the phlegmatic than the melancholy and believes that many individuals Moody people have thoughts about death, which isn't surprising as Beck thinks, because of their emotional state ${ }^{5}$.

In light of the aforementioned, we can visualize a relationship between mood disorders and death anxiety as psychological ailments that the elderly suffer from, and with this we will be preparing for the beginning of the general basis on which this study will generally be conducted, which aims in general to know the level of these two disorders and ensure the existence of the relationship between them and the extent to which they are affected by the personal characteristics of the elderly And, for that, we decided to conduct a field psychological study based on methodological foundations to investigate the truth about these two disorders among this segment of society and residents of some nursing homes for elderly people in Baghdad.

Problems of the study: Demographic statistics indicate, through the researcher's briefing of studies and literature in the field of aging, that the current century will witness an increase in the number of elderly people in societies and nations as a result of the progress of human medicine and the improvement of living conditions. A hundred million people are believed to form (14\%) of the world's population.

The elderly, as a result of the sensitivity of the age stage in which they are going through, and the accompanying general psychological tension and mood disturbance due to physiological changes and deficiencies in mental functions, we find that they suffer from some psychological disorders.

Hence, we chose two types of these disorders, the first of which is mood disorders (affective), which affect the elderly in a large way. Death, the importance of which is evident in its effect on the individual's psychological and physical health, it is expected that the older he approaches the end of life, he becomes more anxious about death.

It is worth noting that the researcher, through his review of the studies and literature of the subject of the research, found that most of them focused on studying one of the two variables without the other and neglected the connection between them, and this is what made us look to the problematic of our current study by examining the relationship between these two disorders.

\section{Research Objectives:}

1. Identifying mood disorders and death anxiety in some elderly homes in Baghdad.

2. Knowing the relationship between mood disorders and death anxiety in some elderly homes in Baghdad.

3. Detecting the level of mood disorders and death anxiety in the elderly, which are useful in judging whether was the level of these disorders moderate or did they reach a level that would pose a threat to the health of the elderly.

\section{Hypothesis:}

1. Most elderly people living in nursing homes have a high level of mood disorders

2. Most elderly people in nursing homes suffer from a high level of death anxiety

3. There is a significant relationship between mood disorders and death anxiety in the sample

\section{1-5 Limits:}

1. Human limits: the infirm and elderly in care homes

2. Place limits : the role of the elderly in Baghdad governorate

3. Temporal limits (2019-2020)

\section{Terminology:}

Mood disorders: This is a disorder that includes alternating symptoms of euphoria, euphoria and hyperactivity mental and physical illness, and darkness 
and mental and physical dullness, and based on that the definition the theoretical concept of mood disorders in this study is a mental or sensory state experienced by the individual in mood swings that oscillate between acute euphoria (obsession) and severe sadness (depression).

Death anxiety: It is defined as a type of generalized anxiety that is not worsening or free and that centers around issues related to death and dying in a person or his family.

\section{Section Three:}

\section{Research methodology and field procedures}

\section{Methodology}

The method used in this study is the descriptive approach, since the majority of researchers are unanimous in considering it an approach that aims to describe the phenomenon under study, diagnose it, shed light on its various aspects and collect the necessary data on it with understanding and analysis in order to reach the principles and laws related to the phenomena of life.

It also depends on the study of the phenomenon as it is in reality as an accurate description and expresses it in a qualitative and quantitative expression, and it is still the most used in human studies so far.

In order to determine whether there is a relationship between two or more quantifiable variables, expressed by the correlation coefficient, the researcher will use the descriptive-correlative approach to determine the relationship between the study variables.

We can notice from the above table that the members of the study sample are distributed as follows:

The total number of the infirmary in the Sulaikh infirmary is (185) males only, and the total number of the infirmary in the Rashad care home is (121) individuals who are females only, while the total number of the infirmary in the al-Kadhimiya infirmary is (25) individuals, consisting of (17) males and (8) females The sample «is that part of the community that is selected according to rules and method so that the community is properly represented.

The sample of the study consisted of (110) elderly people who were able to respond to the researcher, including (65) males and (45) females. Simple randomness,

\begin{tabular}{|l|c|c|c|}
\hline Infirmary & Male & Female & Total \\
\hline Al- Sulaikh Infirmary & 57 & 0 & 57 \\
\hline Al- Rashad Infirmary & 0 & 42 & 42 \\
\hline Al- Kadhimyia Infirmary & 8 & 3 & 11 \\
\hline Total & 65 & 45 & 110 \\
\hline
\end{tabular}

We can notice from the above table that the members of the study sample are distributed as follows:

The number of elderly people in the Al-Sulaikh care home reached (57) males, while the number of people residing in the Rashad care home was (42) females, while the Kadhimiya care home reached (11) individuals

\section{Research instruments:}

Mood disorder scale test: This scale consists of (18) items distributed on two axes: mood rate, and this axis includes the following items $(2,4,6,8,10,11,13$, $15,16)$ and the intensity of the reaction towards postures and includes the following items, 3,5,7,9,12,14,17,18) and this measure was applied.

- Validity of the scale

- Stability of scale

\section{Death anxiety scale:}

- Validity of the scale

- Stability of scale

\section{The statistical means used}

Frequency and percentages to describe the personal characteristics of the study sample

- Calculating arithmetic averages to find out how similar the behavior of the sample members is, as well as calculating the standard deviation to know the nature of the sample members and their harmony.

- $\mathrm{Ca}^{2}$ calculation for classification of mood disorders and death anxiety.

- Pearson correlation coefficient to study the relationship between mood disorders and death anxiety.

\section{Results Preview and Discussion}

This chapter aims to present the results as produced by the statistical treatments of the data obtained after applying the two measures to the studied sample, and the main purpose of the study was to reveal the extent 
of the relationship of mood disorders with death anxiety in the elderly in nursing homes for elderly people in the capital, Baghdad, with knowledge of their average levels and ended The study is to discuss and try to answer the proposed hypotheses, which are as follows:

Results of the first hypothesis: The majority of elderly people residing in nursing homes suffer from a high level of mood disorders.
To answer this hypothesis, and after using some statistical method, the level of mood disorder among elderly sample members in elderly care homes in the capital Baghdad was determined, and the difference in levels was extracted by calculating:

$\mathrm{Ca}^{2}=\left(\mathrm{x}^{2}=32.58\right)$ in a free degree $(02)$ in the indication level of (0.01) and the following table clarify that.

\begin{tabular}{|l|c|c|c|c|c|c|c|}
\hline The scale & Repetition & Percentage & Term & Level & \multirow{2}{*}{$\mathbf{C a}^{\mathbf{2}}$} & Free degree $^{\text {Indication level }}$ \\
\hline \multirow{4}{*}{ Mood disorders } & 10 & $9.1 \%$ & $18 \_48$ & Low & & & \\
\cline { 2 - 6 } & 42 & $38.2 \%$ & $48.1 \_78$ & Medium & \multirow{2}{*}{32.85} & 2 & \begin{tabular}{c} 
Indicates in level \\
\cline { 2 - 6 }
\end{tabular} \\
\cline { 2 - 6 } Total & 58 & $52.7 \%$ & $78.1 \_108$ & High & & & \\
\hline
\end{tabular}

It is evident from the above table that the majority of the sample members obtained a high level of mood disorder where the frequency of (58) members of the study sample $(110=\mathrm{N})$ was at this level, and depending on the criteria adopted in this study, their frequency falls within the range (87.1_108) Therefore, the level of mood disorders of the subjects of the study was classified as high and close to the average, and as the table shows, $(52.7 \%)$ of the study sample individuals represented this level of mood disorders, while it had (38.2\%) The sample members had a medium level of disorders, and only $(9.1 \%)$ of them had a low level of mood disorders and at a level of significance (0.01).

\section{The second hypothesis results:}

ost of elderly who live in the infirmaries are suffering from death anxiety

The table shows the distribution of the sample members according to levels on the death anxiety scale

\begin{tabular}{|l|c|c|c|c|c|c|c|}
\hline Scale & Repetition & Percentage & Term & Level & \multirow{2}{*}{$\mathbf{C a}^{\mathbf{2}}$} & Free degree & Indication level \\
\hline \multirow{3}{*}{ Death anxiety } & 16 & $14.5 \%$ & $0 \_6$ & Low & \multirow{2}{*}{39.29} & \multirow{2}{*}{2} & \\
\cline { 2 - 6 } & 67 & $60.9 \%$ & $7 \_14$ & Medium & \multirow{2}{*}{ Not indicated } \\
\cline { 2 - 7 } & 27 & $24.5 \%$ & $15 \_20$ & High & & & \\
\hline Total & $\mathbf{1 1 0}$ & $\mathbf{1 0 0} \%$ & & & & & \\
\hline
\end{tabular}

The results of the current study do not support this hypothesis, when determining levels of death anxiety counts.

$\mathrm{Ca}^{2}=\left(39.29 \_2 \mathrm{X}\right)$ and the degree of freedom (02) at a level of significance (0.01) for the study sample. It appeared that the majority of the individuals suffer from a moderate level of death anxiety, and this means that the result did not obtain the statistical significance as it was the repeat of (67) individuals out of a total The sample members $(\mathrm{N}=110)$ have this level of anxiety and that this level falls within the range (7_14). Therefore, the level of death anxiety was classified on the anxiety scale as medium. Death anxiety while $(24.5 \%)$ of them represented the high level of death anxiety and only $(14.5 \%)$ of them had the low level of death anxiety.

It is possible that the scale used in this study includes items with clear negativity, which made the respondents' answers unrealistic, and also perhaps due to the fact that some elderly people lived through the death cases that occurred in recent days, which made them not care about death and are not afraid of it to a great extent. It remains for us to note that correlational relationships Between the total degree of death anxiety and the scores on the paths of death anxiety was a function of all domains, 
and for both genders, the highest correlation was (0.64) between the total degree of death anxiety and the degree on the field of pain and suffering, and the relationship was significant at the level (0.01).

Third Hypothesis Results: There is a significant relationship between mood disorders and death anxiety among the sample of elderly people residing in hospice homes in the capital, Baghdad. This hypothesis was not supported, which is considered a major hypothesis in our current study. Mood and death anxiety are weak and the correlation value was (0.06) according to the following table for the value of the correlation coefficient.

Explains the link between mood disorders and death anxiety and notes the relationship It is (0.06), and it is a non-statistically significant relationship

\begin{tabular}{|l|c|c|}
\hline & Mood disorders & Death anxiety \\
\hline Mood disorders Pearson correlation Sig(2-tailed) & 1.00 & 0.06 \\
N & & 0.84 \\
& 110 & 110 \\
\hline Death anxiety Pearson correlation Sig(2-tailed) & 0.06 & 1.00 \\
N & 0.84 & 110 \\
\hline
\end{tabular}

This result needs a lot of reflection in order to find an appropriate explanation for it, because the psychological heritage related to mood disorder contains important signals that push towards the expectation of a high rate of disturbance, and at the same time, some studies related to death anxiety showed that whatever increases the individual's life, the fear of death becomes more. Urgent, comprehensive, and continuous, hence it was expected that the two variables correlation among the sample members would be statistically significant.

And if we try to explain the lack of the relationship, it may be due to a number of reasons, including the size of the studied sample, which was small in this study, which affected the lack of a statistically related relationship between these two variables, in addition to the inaccuracy in choosing the subjects who suffer from high psychological disorders because the choice came randomly.

\section{Conclusions and Recommendations}

1. The suffering of the elderly living in nursing homes in the capital, Baghdad, from a high level of mood disorders.

2. The suffering of the elderly residing in the nursing homes of the capital, Baghdad, from a moderate feeling of anxiety in death.

3. The results show that mood disorders and death anxiety were found in females and males.
4. The necessity of conducting new studies dealing with the variables of the current study, whether each of them is taken separately or with other variables, in order to make more sure of the results of this study.

5. It is better to use multiple method of measurement and not to be satisfied with one method for each variable. Perhaps it is possible to use more positive measurement method.

6. Developing programs to help workers in care homes understand psychological disorders and general anxiety, including in particular death anxiety.

7. The necessity for individuals to be educated to respect and appreciate the elderly as a humanitarian duty and imposed on us by our religious and Islamic human values.

8. Establishing a network of social contacts and emotional support for the elderly internally and externally, such as establishing and improving their relationships within the care homes themselves.

Financial Disclosure: There is no financial disclosure.

Conflict of Interest: None to declare.

Ethical Clearance: All experimental protocols were approved under The General Directorate of DhiQar Education and all experiments were carried out in accordance with approved guidelines. 


\section{References}

1. Abd al-Khaleq A. The Basic Dimensions of the Personality, House of Knowledge University, Egypt, 1983.

2. Abdul Khaleq A. Anxiety of Death, The World of Knowledge Series, Kuwait, 1987.

3. Al-Hanafi A. Comprehensive Dictionary of Philosophy Terms, 2nd Edition, Library, Madbouly, Cairo, 2000.

4. Khalifa IA. Utilizing the full capabilities of the elderly to care for children in the Arab world,, The Arab Future Magazine, Volume/7 Issue/67, Center for Arab Unity Studies, Beirut, 1984.

5. Lonett R. the Nature of death an xiety, gnic. D.spiel Berger and but cher (Eds), Advances in personality assessment, hillsdaie, New Jersey, Lawrence, Erlbaum, Associates, publishers, No. 3, 1983.
6. Al-Samarrai A. Some psychological disorders and their impact on committing crime, Journal of the College of Education, Al-Mustansiriya University, Baghdad, 1997.

7. Abdul Khaleq AM. A source previously mentioned, 1987 .

8. Shafiq M. Scientific Research, Methodological Steps for Preparing Social Research, Modern University Office, Alexandria, 1998.

9. Bouhouch A, Al-Thunaibat M. Scientific Research Method, University Press Office, Algeria, 1984.

10. Jay: Educational Research Skills, 2nd Edition, Arab Renaissance House, Qatar, 1993, p. 296.

11. Jamal Z. Foundations of Social Research, Cairo, Arab Thought House, 1962. 